\title{
Los polifenoles de la fresa disminuyen el estrés oxidativo en enfermedades crónicas
}

\author{
Cecilia Isabel Oviedo-Solís', Sinthia Cornejo-Manzo², Blanca Olivia Murillo-Ortiz³, Michelle Montserrat \\ Guzmán-Barrón ${ }^{4}$ y Joel Ramírez-Emiliano ${ }^{5}$
}

${ }^{1}$ Maestría en Investigación Clínica, Universidad de Guanajuato; 'Licenciatura en Nutrición, Universidad Insurgentes; ${ }^{3}$ Unidad Médica de Alta Especialidad No. 1 Bajío, IMSS; ${ }^{4}$ Licenciatura en Químico Farmacéutico Biólogo; ${ }^{5}$ Departamento de Ciencias Médicas, Universidad de Guanajuato. León, Gto., México

\begin{abstract}
Resumen
El consumo de dietas hipercalóricas conlleva al aumento de ácidos grasos libres (AGL), citocinas proinflamatorias y producción de especies reactivas de oxígeno y de nitrógeno. Estas alteraciones inducen estrés oxidativo y nitrosativo que daña a los tejidos causando disfunción de los mismos y en consecuencia se pueden desarrollar enfermedades crónicas. Por lo tanto, es importante disminuir el estrés oxidativo y con ello prevenir el desarrollo de estas enfermedades. La fresa es un fruto rico en vitamina $C$ y polifenoles, compuestos con excelentes propiedades antioxidantes, por lo que puede ser una opción para la disminución del estrés oxidativo y por lo tanto, para prevenir el desarrollo de algunas enfermedades. Los estudios realizados in vitro, en modelos animales y estudios clínicos sustentan que la fresa puede ser una buena alternativa para disminuir el estrés oxidativo y así atenuar y/o prevenir el desarrollo de enfermedades en el humano.
\end{abstract}

PALABRAS CLAVE: Dieta hipercalórica. Obesidad. Estrés oxidativo. Fresa. Polifenoles.

\begin{abstract}
Consumption of hypercaloric diets leads to increase of free fatty acids (FFA), pro-inflammatory cytokines and production of oxygen and nitrogen reactive species. These alterations induce oxidative and nitrosative stress causing dysfunction of tissues and consequently the development of chronic diseases. Therefore, it is important to decrease oxidative stress and thus preventing the development of these diseases. Strawberry has a lot of Vitamin $C$ and polyphenols, compounds with excellent antioxidant properties, which may be an option for reducing oxidative stress and therefore to prevent the development of some diseases. Studies conducted in vitro in animal models and clinical studies support that this fruit can be a good alternative to reduce oxidative stress and thus reducing and/or preventing the development of diseases in humans.
\end{abstract}

KEY WORDS: Hypercaloric diet. Obesity. Oxidative stress. Strawberry. Polyphenols.

\author{
Correspondencia: \\ Joel Ramírez-Emiliano \\ Departamento de Ciencias Médicas \\ División de Ciencias de la Salud \\ Campus León, Universidad de Guanajuato \\ 20 de Enero, 929 \\ Col. Obregón \\ C.P. 37320, León, Gto. México \\ E-mail: joelre@ugto.mx
}

\author{
Fecha de recepción en versión modificada: 12-07-2016 \\ Fecha de aceptación: 28-11-2016 \\ DOI://dx.doi.org/10.24875/GMM.17002759
}

Gac Med Mex. 2018;154:80-86

Contents available at PubMed www.gacetamedicademexico.com 


\section{Introducción}

En México la Encuesta Nacional de Salud y Nutrición 2012 mostró que la prevalencia de sobrepeso y obesidad es del $71.3 \%$ para ambos géneros, lo que corresponde a un $73 \%$ en mujeres y un $69.4 \%$ en hombre. También reportó que los costos por obesidad han sido estimados en 67 mil millones de pesos en el 2008', por lo que es urgente tomar las medidas adecuadas para disminuir el aumento de la obesidad.

A pesar de las particularidades de la dieta de los mexicanos, esta es muy semejante a la dieta occidental, es hipercalórica, con alto contenido en grasa y carbohidratos, por lo que es uno de los factores determinantes en el desarrollo de la obesidad. Evidencias apoyan que la dieta hipercalórica con alto contenido en grasa y carbohidratos contribuye significativamente al desarrollo de la obesidad y síndrome metabólico en animales ${ }^{2}$ y en humanos ${ }^{3}$. El síndrome metabólico es un grupo de enfermedades cronicometabólicas que incluyen hipertensión, dislipidemias, resistencia a la insulina, obesidad y diabetes ${ }^{4}$. Así, las dietas hipercalóricas pueden inducir un estado inflamatorio y mayor producción de radicales libres (RL) (Fig. 1), lo que trae como consecuencia estrés oxidativo y el desarrollo de enfermedades cronicometabólicas, como se revisará más adelante.

\section{Radicales libres y sistemas antioxidantes}

Los RL son especies químicas con un electrón desapareado en su orbital más externo, esto les da una configuración inestable y, por lo tanto, una gran capacidad de reaccionar con otras moléculas. Los $\mathrm{RL}$ se dividen en especies reactivas derivadas del nitrógeno (ERN) y especies reactivas derivadas del oxígeno $(E R O)^{5}$. Los $\mathrm{RL}$ se producen fisiológicamente en algunas reacciones llevadas a cabo como parte del metabolismo, por ejemplo, en la mitocondria de mamíferos en estado 4 , del 1 al $2 \%$ del oxígeno consumido es convertido en ERO ${ }^{6-8}$. Cabe mencionar que la producción controlada de $\mathrm{RL}$ permite que se lleven a cabo diversos procesos fisiológicos como la neurotransmisión, la vasorrelajación, la fertilización del óvulo por el espermatozoide, la activación de genes y enzimas de la membrana celular, la síntesis de colágeno, lisis de bacterias, etc., pero la producción excesiva de $R L$ es dañina para fisiología celular.
Las ERO y las ERN son descompuestas y/o neutralizadas por antioxidantes exógenos y endógenos. Los antioxidantes exógenos son hidrosolubles (ácido ascórbico), liposolubles (tocoferoles, carotenoides, xantofilas) o con ambas propiedades (polifenoles, flavonoides, taninos, ligninas, fenilpropanoides). Por su parte, los antioxidantes endógenos de tipo enzimático son la superóxido dismutasa dependiente de manganeso (MnSOD) y la coenzima $Q(C o-Q)$, que se encuentran en el espacio intermembranal de la mitocondria, además de la superóxido dismutasa (SOD) dependiente de cobre (CUSOD), la SOD dependiente de zinc (ZnSOD), la glutatión peroxidasa (GSH-Px), la fosfolípido hidroperóxido glutatión peroxidasa $(\mathrm{PH}-$ GPX) y las catalasas que se encuentran en el citosol9-11. $^{9}$.

El estrés oxidativo ocurre cuando la producción de $\mathrm{RL}$ rebasa la capacidad de los sistemas antioxidantes exógenos y endógenos ${ }^{12-14}$. Así, el incremento de RL tiene efectos tóxicos en las células y tejidos debido a que pueden oxidar carbohidratos, DNA, lípidos y proteínas; siendo un importante mecanismo en el desarrollo de enfermedades cronicometabólicas (véase Fig. 1) como se revisará más adelante.

\section{Dieta hipercalórica, estrés oxidativo y disfunción de órganos}

Utilizando modelos murinos se demostró que las dietas hipercalóricas con alto contenido en grasa y/o carbohidratos inducen incremento de tejido adiposo que conlleva aumento de peso corporal $\left.\right|^{2,15-17}$, mientras que en humanos se asociaron fuertemente las dietas hipercalóricas con el aumento de peso corporal ${ }^{18-20} \mathrm{y}$, por otro lado, el sobrepeso y/o obesidad con el aumento de citocinas proinflamatorias ${ }^{21-23}$. En modelos animales está bien documentado que la dieta hipercalórica causa aumento de peso y mayor producción de citocinas proinflamatorias (Fig. 1). Por ejemplo, la dieta hipercalórica aumentó la producción de interleucina (IL) 1, IL-6 y factor de necrosis tumoral $\alpha$ (TNF- $\alpha$ ); también indujo mayor producción de RL y estrés oxidativo ${ }^{15,17,24-26}$. Además, la dieta hipercalórica indujo obesidad y el desarrollo de esteatosis hepática en modelos animales ${ }^{2,16}$. En humanos únicamente se ha relacionado la dieta hipercalórica con la obesidad y con el contenido de ácidos grasos en hígado, que fueron medidos por ultrasonografía ${ }^{19}$.

El consumo de dietas hipercalóricas con alto contenido en AGL es preocupante, debido a que estos incrementan la producción de ERO (Fig. 1). En ratas, 


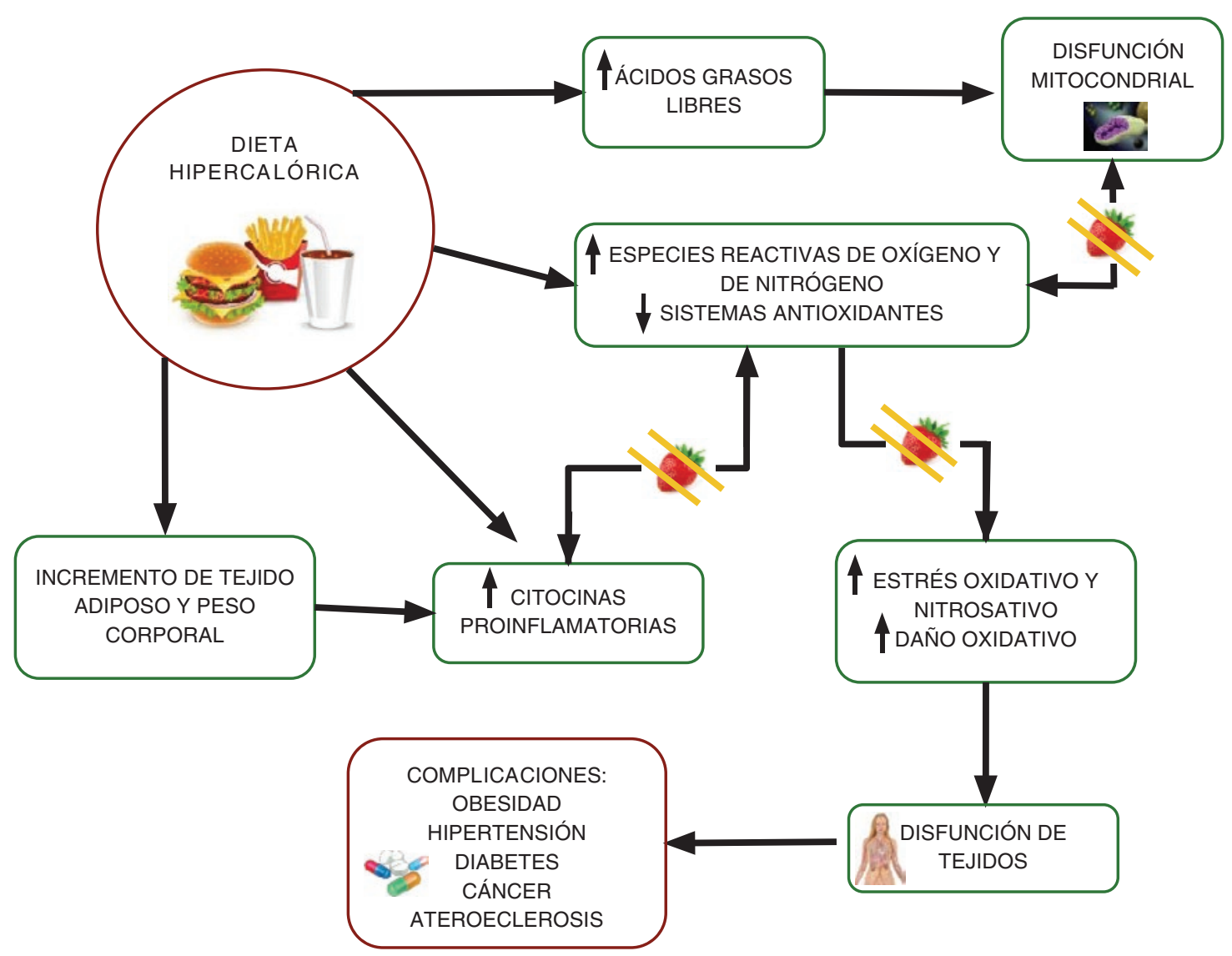

Figura 1. Modelo hipotético de disfunción de tejidos e inducción de enfermedades crónicas por las dietas hipercalóricas. Las dietas hipercalóricas inducen el desarrollo de enfermedades crónicas, mientras que los polifenoles de la fresa pueden prevenir el desarrollo de estas enfermedades mediante la disminución del estrés oxidativo y nitrosativo.

el aumento de los niveles plasmáticos de AGL incrementó la producción de ERO27. Los AGL también incrementaron la producción de ERO en células endoteliales de aorta y en células- $\beta$ humanas in vitro ${ }^{28,29}$. En células neuronales in vitro, los AGL incrementaron la producción de ERO y la peroxidación de lípidos, esto desacopló la mitocondria ${ }^{29}$. Similarmente, en células de hepatoma humano in vitro, los AGL desacoplaron la mitocondria e incrementaron el estrés nitrosativo y oxidativo, reduciendo la fosforilación oxidativa ${ }^{30}$. Los AGL también indujeron la producción de IL-1 $\alpha$ y el desacoplamiento mitocondrial en macrófagos in vitro ${ }^{31}$. Además, la sacarosa incrementa la producción de ERO y la peroxidación de lípidos en aorta aislada de ratas ${ }^{32}$. Todos estos estudios muestran que las dietas hipercalóricas ricas en lípidos y/o carbohidratos contribuyen significativamente al desarrollo del estado inflamatorio, producción de $R L$ y obesidad.

En humanos, las dietas hipocalóricas disminuyen el peso corporal, la secreción de leptina, proteína $\mathrm{C}$ reactiva, TNF- $\alpha$, IL-6 $6^{33-35}$ y los marcadores de lípidos oxidados ${ }^{36}$, mientras que la disminución de peso corporal disminuyo la resistencia a la insulina y los niveles de lipoproteínas de baja densidad (LDL) oxidadas $^{37}$. Estos datos refuerzan los datos obtenidos en modelos animales, en el sentido que las dietas hipercalóricas inducen la expresión de citocinas proinflamatorias y la producción RL, lo que contribuye al desarrollo de enfermedades como la diabetes.

Con base en lo descrito anteriormente, como se muestra en la figura 1, es muy probable que las dietas hipercalóricas induzcan estrés y daño oxidativo de dos maneras. Primero, la dieta hipercalórica incrementa la producción de citocinas proinflamatorias, las cuales sobreestimulan a las células para incrementar la producción de ERO y ERN. Segundo, las dietas hipercalóricas inducen desacoplamiento mitocondrial por el incremento de AGL, lo que conlleva una mayor producción de ERO por la misma mitocondria. Sin embargo, no está claro si las dietas hipercalóricas primero incrementan la producción de citocinas proinflamatorias o si primero desacoplan la mitocondria, o si ambos procesos son producidos simultáneamente. Independientemente de 
cuál proceso se lleva a cabo primero, el resultado es la presencia de estrés oxidativo que conlleva un incremento de la oxidación de macromoléculas importantes para la fisiología celular.

La oxidación de macromoléculas trae como consecuencia el desarrollo de complicaciones tales como la diabetes. Por ejemplo, Carvalho-Filho, et al., demostraron en 2005 que en ratas, la dieta rica en grasa induce nitración del receptor de insulina, del sustrato del receptor a insulina y de la proteína cinasa B/Akt, lo que condujo al desarrollo de la resistencia a la insulina $^{38}$. Además, en ratones, la dieta rica en grasa dañó la captación de glucosa en el músculo, esta resistencia a insulina se acompañó de sobreexpresión de la NADPH (nicotinamide adenine dinucleotide phosphate) oxidasa 2 y mayor liberación de peróxido de hidrógeno. También indujo la disminución de la proporción glutatión reducido/oxidado (GSH/GSSG) ${ }^{39}$, sistema antioxidante muy importe para la célula.

\section{Uso de la fresa en estudios clínicos}

Es evidente la necesidad de disminuir el estrés oxidativo y con ello prevenir y/o retardar el desarrollo de enfermedades cronicometabólicas. Al respecto, los alimentos ricos en antioxidantes pueden disminuir el estrés oxidativo, para ello es deseable que estos posean gran reactividad hacia los RL; también que los antioxidantes sean liposolubles, para que pueda atravesar las membranas biológicas y neutralicen los RL, y tengan la capacidad de neutralizar la presencia de reacciones secundarias, es decir, neutralizar los $R L$ secundarios ${ }^{12,40}$.

En los últimos años se ha despertado el interés por los polifenoles debido a su alta capacidad antioxidante, por lo que tienen un gran potencial en la prevención y/o tratamiento de diversas enfermedades en las cuales el estrés oxidativo tiene un efecto significativo en la fisiopatología de la enfermedad (Fig. 1). Los polifenoles se encuentran ampliamente distribuidos en alimentos de origen vegetal como la cúrcuma, las espinacas, la uva, la fresa, la manzana, los arándanos, la granada y el cacao, entre otros. En esta revisión nos centraremos en la fresa por sus propiedades antioxidantes y su potencial en la salud.

La fresa tiene gran contenido de compuestos fenólicos como antocianinas (pelargonidina, etc.), flavonoles (quercetina, etc.), proantocianidinas (procianidina, etc.), elagitaninos (agrimoniin, etc.), glucósidos del ácido elágico y conjugados del ácido cinámico (hexosa coumaroil y glucosa cinamoil) ${ }^{41,42}$. La fresa también es de gran interés por su alto contenido de vitamina $\mathrm{C}$, que junto con los polifenoles le confieren un gran poder antioxidante que puede ser benéfico para la salud, potencial que ha sido evidenciado en diversas investigaciones, como se discute a continuación.

En un estudio, voluntarios sanos consumieron 500 $\mathrm{g}$ de fresas/día durante un mes. La fresa redujo significativamente el colesterol total, las LDL y los triglicéridos, es decir, mejoró el perfil de lípidos plasmático. También disminuyó significativamente los niveles de malondialdehído (MDA) en suero y orina, y mejoró los biomarcadores de estado antioxidante, las defensas antihemolíticas y la función plaquetaria ${ }^{43}$. En otro estudio realizado en hombres y mujeres aparentemente sanos, consumieron durante dos semanas $500 \mathrm{~g}$ de fresas/día por cada $70 \mathrm{~kg}$ de peso corporal. Al final del tratamiento, se observó un incremento moderado de vitamina $\mathrm{C}$ y de la capacidad antioxidante del plasma en ayunas, además de retardo en la oxidación de los lípidos plasmáticos y aumento en la resistencia a la hemólisis oxidativa de eritrocitos ${ }^{44}$. En mujeres adultas mayores sanas se evaluó cuánto se incrementa la capacidad antioxidante del suero al consumir alguno de los siguientes antioxidantes: $240 \mathrm{~g}$ de fresas, $294 \mathrm{~g}$ de espinacas, $300 \mathrm{ml}$ de vino tinto, $125 \mathrm{mg}$ de vitamina $\mathrm{C}$ o bebida control. Los resultados mostraron que la capacidad antioxidante total del suero aumentó significativamente del 7 al 25\% durante el periodo de $4 \mathrm{~h}$ tras el consumo de vino tinto, fresas, vitamina $\mathrm{C}$ o espinacas. También la capacidad antioxidante en orina aumentó un 9.6, 27.5 y $44.9 \%$ en aquellos que consumieron fresas, espinacas y vitamina $\mathrm{C}$, respectivamente, durante el periodo de $24 \mathrm{~h}$ después de estos tratamientos ${ }^{45}$.

En un estudio cruzado, hombres y mujeres adultos con sobrepeso con consumo habitual de alimentos con alto contenido en carbohidratos y moderado contenido graso ingirieron una bebida con $10 \mathrm{~g}$ de fresa liofilizada y placebo. La bebida con fresa incrementó los niveles de antocianinas y sus metabolitos en el plasma en el estado pospandrial, mientras que disminuyó los marcadores inflamatorios como la PCR y la IL-6; también se asoció con una mejora en la sensibilidad a la insulina ${ }^{46}$. En hombres y mujeres con hiperlipidemia que consumieron $10 \mathrm{~g}$ de liofilizado de fresa en bebida después de una comida alta en grasa, los niveles de triglicéridos y las LDL oxidados disminuyeron significativamente después de seis semanas, en comparación con el grupo que consumió placebo ${ }^{47}$. En un estudio doble ciego en el cual participaron 20 adultos obesos de ambos géneros, estos consumieron 
liofilizado de fresa (equivalente a 4 porciones de fresa congelada) durante tres semanas. Al final del tratamiento, disminuyeron la concentración plasmática de colesterol y las pequeñas partículas de colesterol de alta densidad (HDL), y aumentó el tamaño de las partículas de LDL, cambios asociados a la disminución de riesgo cardiovascular ${ }^{48}$.

Sin embargo, en hombres y mujeres que consumieron $25 \circ 50 \mathrm{~g}$ de fresa/día durante 12 semanas no se observó efecto sobre las medidas de adiposidad, presión arterial y glucemia, y tampoco hubo efecto sobre las concentraciones séricas de $\mathrm{HDL}$, los triglicéridos y la PCR. El grupo que consumió $50 \mathrm{~g}$ de fresa únicamente mostró reducción del colesterol LDL en comparación con la dosis baja de fresa. Ambas cantidades de fresa mostraron disminución de MDA en suero $^{49}$. Igualmente, a hombres y mujeres obesos con una dieta alta en hidratos de carbono y grasa se les administró durante seis semanas $305 \mathrm{~g}$ de bebida de fresa o placebo $(10 \mathrm{~g}$ y $0 \mathrm{~g}$ de fresa liofilizada, respectivamente). El grupo que consumió bebida de fresa mostró atenuación significativa de las concentraciones posprandiales del inhibidor del activador del plasminógeno-1 (PAI-1) y disminución no significativa de IL-1 $\beta$. No se encontró diferencia en la agregación plaquetaria, la prueba de PCR de alta sensibilidad, el TNF- $\alpha$, la insulina o la glucosa ${ }^{50}$.

En un estudio de cohorte donde se incluyeron 16,010 participantes enfermeros, con seguimiento de cuatro años, se encontró que un mayor consumo de arándanos y fresas se asocia a una tasa más baja de deterioro cognitivo; además parece retrasar el envejecimiento cognitivo hasta 2.5 años ${ }^{51}$. Estos resultados ponen de manifiesto que una dieta rica en compuestos fenólicos tiene el potencial de limitar la neurodegeneración y el declive cognitivo $0^{52,53}$ y se asocia a un menor riesgo de infarto de miocardio ${ }^{54}$.

\section{Uso de la fresa en modelos animales}

Más puntualmente, en modelos animales también se ha demostrado la efectividad de la fresa para disminuir el estrés oxidativo. Por ejemplo, en ratas macho que fueron alimentadas con aceite de fresa y de frambuesa durante cinco semanas, disminuyó la actividad de la SOD y la glutation peroxidasa, (GSH-Px) lo que sugiere que los aceites disminuyeron o eliminaron los RL, por lo que se redujo la actividad de estas enzimas antioxidantes. Sin embargo, no resultaron afectados los niveles de triglicéridos, colesterol total, LDL, ni el colesterol HDL ${ }^{55}$. En otra investigación, la suplementación de fresa a ratas durante dos meses redujo significativamente la producción de ERO y el daño al ADN inducido por DOX (doxorubicina), así como mejoró los biomarcadores de estrés oxidativo, las actividades enzimáticas antioxidantes y el rendimiento mitocondrial. Este trabajo confirmó que la suplementación de la fresa puede contrarrestar el estrés oxidativo causado por DOX ${ }^{56}$. En ratas albinas con enfermedad inflamatoria del intestino, el extracto etanólico de fresa mejoró el cuadro de la enfermedad y se considera que fue por las propiedades antioxidantes e inflamatorias del extracto ${ }^{57}$, además se observó reducción de la actividad $\beta$-glucuronidasa en el ciego y en las heces, lo que indica cambios positivos en la microbiota de las ratas $^{58}$. Por su parte, Casto, et al. demostraron que las fresas liofilizadas pueden inhibir la formación de tumores en la cavidad oral del hámster ${ }^{59}$. Dudonné (2014) mostró que hay actividad sinérgica de los compuestos fenólicos de la fresa con la quercetina de la cebolla, porque juntos mejoraron la biodisponibilidad, con una posible inducción intestinal de la UDP-glucuronosil-transferasa. Tres compuestos conjugados glucurónidos de compuestos fenólicos de fresa y arándanos (ácido p-hidroxibenzoico glucurónido, catequinas glucurónidos y metil-catequina glucurónido) se encontraron en mayores cantidades cuando fueron ingeridos con la cebolla ${ }^{60}$.

Interesantemente, un estudio demostró que la capacidad antioxidante de la fresa también se puede observar en la conservación de los alimentos. Un estudio añadió el extracto de fresa a empanadas de pollo que fueron almacenadas en refrigeración durante seis días. El extracto de fresa disminuyó la oxidación lipídica, lo que demuestra que el extracto tiene un buen poder antioxidante y previene la oxidación de los alimentos ${ }^{61}$.

\section{Conclusiones}

Las evidencias abordadas en esta revisión sustentan que las dietas hipercalóricas pueden inducir un estado proinflamatorio, caracterizado por incremento de AGL, citocinas proinflamatorias y producción de $\mathrm{RL}$, contribuyendo al desarrollo de patologías relacionadas con la alteración del estado redox celular. También sustenta que la fresa, debido a su elevado contenido de sustancias antioxidantes, puede atenuar el estrés oxidativo y/o prevenir el desarrollo de enfermedades en el humano.

\section{Agradecimientos}

Este trabajo fue realizado durante el apoyo otorgado por CONACYT (1010/532/2014) y la Universidad de 
Guanajuato (528/2015) a J. R-E. C. I. O-S recibió beca de CONACYT para estudios de Maestría (589934).

\section{Bibliografía}

1. Gutiérrez J, Rivera-Dommarco J, Shamah-Levy T, et al. Encuesta Nacional de Salud y Nutrición 2012. Resultados nacionales. Cuernavaca, México: Instituto Nacional de Salud Pública; 2012.

2. Yang ZH, Miyahara $\mathrm{H}$, Takeo J, et al. Diet high in fat and sucrose induces rapid onset of obesity-related metabolic syndrome partly through rapid response of genes involved in lipogenesis, insulin signalling and inflammation in mice. Diabetol Metab Syndr. 2012;4:32.

3. Bruce K, Hanson M. The developmental origins, mechanisms, and implications of metabolic syndrome. J Nutr. 2010;648-52.

4. Alberti KG, Zimmet P, Shaw J. Metabolic syndrome - a new world-wide definition. A consensus statement from the international diabetes federation. Diabet Med. 2006;23:469-80.

5. Radi R. Nitric oxide, oxidants, and protein tyrosine nitration. Proc Nat Acad Sci U S A. 2004;101:4003-8.

6. Boveris $A$, Chance $B$. The mitochondrial generation of hydrogen peroxide. General properties and effect of hyperbaric oxygen. Biochem J. 1973;134:707-16.

7. Chance B, Sies H, Boveris A. Hydroperoxide metabolism in mammalian organs. Physiol Rev. 1979;59:527-605.

8. Forman HJ, Davies KJ, Ursini F. How do nutritional antioxidants really work: Nucleophilic tone and para-hormesis versus free radical scavenging in vivo. Free Radic Biol Med. 2014;66:24-35.

9. Blokhina O, Virolainen E, Fagerstedt KV. Antioxidants, oxidative damage and oxygen deprivation stress: A review. Ann Bot. 2003:91:179-94

10. Pérez-Vázquez V, Ramírez J, Aguilera-Aguirre L, et al. Effect of Ca2+ and $\mathrm{Mg} 2+$ on the $\mathrm{Mn}$-superoxide dismutase from rat liver and heart mitochondria. Amino Acids. 2002;22:405-16.

11. Kohen R, Nyska A. Oxidation of biological system: oxidative stress phenomena, antioxidants, redox reactions, and methods for their quantification. Toxicol Pathol. 2002;30:620-50.

12. López-Alarcón C, Denicola A. Evaluating the antioxidant capacity of natural products: A review on chemical and cellular-based assays. Anal Chim Acta. 2013;763:1-10

13. Lobo V, Patil A, Phatak A, et al. Free radicals, antioxidants and functional foods: Impact on human health. Pharmacogn Rev. 2010;4:118-26.

14. Akram M. Flavonoids and phenolic acids: Role and biochemical activity in plants and human. J Med Plants Res. 2011;5:6697-703.

15. Martínez-Morúa A, Soto-Urquieta MG, Franco-Robles E, et al. Curcumin decreases oxidative stress in mitochondria isolated from liver and kidneys of high-fat diet-induced obese mice. J Asian Nat Prod Res. 2013;15:905-15.

16. Podrini C, Cambridge EL, Lelliott CJ, et al. High-fat feeding rapidly induces obesity and lipid derangements in C57BL/6N mice. Mamm Genome. 2013;24:240-51.

17. Waise TMZ, Toshinai K, Naznin F, et al. One-day high-fat diet induces inflammation in the nodose ganglion and hypothalamus of mice. Biochem Biophys Res Commun. 2015;464:1157-62.

18. Worobey J, Trytko U. Associations between maternal feeding style and child overweight. ICAN: Infant, Child, \& Adolesc Nutr. 2014;6:216-20.

19. Koopman KE, Caan MW, Nederveen AJ, et al. Hypercaloric diets with increased meal frequency, but not meal size, increase intrahepatic triglycerides: A randomized controlled trial. Hepatology. 2014;60:545-53.

20. Perkins JM, Joy NG, Tate DB, et al. Acute effects of hyperinsulinemia and hyperglycemia on vascular inflammatory biomarkers and endothelia function in overweight and obese humans. Am J Physiol Endocrinol Metab. 2015;309:E168-76.

21. Skinner AC, Steiner MJ, Henderson FW, et al. Multiple markers of inflammation and weight status: cross-sectional analyses throughout childhood. Pediatrics. 2010;125:801-9.

22. Zhang XM, Guo L, Chi MH, et al. Identification of active miRNA and transcription factor regulatory pathways in human obesity-related inflammation. BMC Bioinformatics. 2015;16:1-7.

23. Tucakovic L, Colson N, Singh I. Relationship between common dietary polyphenols and obesity-induced inflammation. Food and Public Health. 2015;5:84-91

24. Charradi K, Elkahoui S, Limam F, et al. High-fat diet induced an oxidative stress in white adipose tissue and disturbed plasma transition metals in rat: Prevention by grape seed and skin extract. J Physiol Sci. 2013:63:445-55.

25. Porto ML, Lírio LM, Dias AT, et al. Increased oxidative stress and apoptosis in peripheral blood mononuclear cells of fructose-fed rats. Toxicol Vitr. 2015;29:1977-81.

26. Han F, Hui Z, Zhang S, et al. Induction of Haemeoxygenase-1 improves FFA-induced endothelial dysfunction in rat aorta. Cell Physiol Biochem. 2015;35:1230-40
27. Pereira S, Shah A, George Fantus I, et al. Effect of N-acetyl-L-cysteine on insulin resistance caused by prolonged free fatty acid elevation. $\mathrm{J}$ Endocrinol. 2015;225:1-7.

28. Yuan $H$, Zhang $X$, Huang $X$, et al. NADPH oxidase 2-derived reactive oxygen species mediate FFAs-induced dysfunction and apoptosis of b-cells via JNK, p38 MAPK and p53 pathways. PLoS One. 2010;5:1-9.

29. Zarrouk A, Nury T, Riedinger JM, et al. Dual effect of docosahexaenoic acid (attenuation or amplification) on C22 : 0 -, C24 : 0-, and C26 : 0-induced mitochondrial dysfunctions and oxidative stress on human neuronal Sk-N-Be cells. J Nutr Health Aging. 2015;19:1-11.

30. Garcia-Ruiz I, Solis-Munoz P, Fernandez-Moreira D, et al. In vitro treatment of HepG2 cells with saturated fatty acids reproduces mitochondrial dysfunction found in nonalcoholic steatohepatitis. Dis Model Mech. 2015;8:183-91.

31. Freigang $S$, Ampenberger $F$, Weiss $A$, et al. Fatty acid-induced mitochondrial uncoupling elicits inflammasome-independent IL-1[alpha] and sterile vascular inflammation in atherosclerosis. Nat Immunol. 2013;14:1045-53.

32. Ruiz-Ramírez A, Ortiz-Balderas E, Cardozo-Saldaña G, et al. Glycine restores glutathione and protects against oxidative stress in vascular tissue from sucrose-fed rats. Clin Sci. 2014;126:19-29.

33. Arvidsson E, Viguerie N, Andersson I, et al. Effects of different hypocaloric diets on protein secretion from adipose tissue of obese women. Diabetes. 2004;53:1966-71

34. Hermsdorff HH, Zulet MÁ, Abete I, et al. A legume-based hypocaloric diet reduces proinflammatory status and improves metabolic features in overweight/obese subjects. Eur J Nutr. 2011;50:61-9.

35. Iriyama $\mathrm{Y}$, Murayama N. Effects of a worksite weight-control programme in obese male workers: A randomized controlled crossover trial. Health Educ J. 2014;73:247-61.

36. Crujeiras AB, Parra D, Goyenechea E, et al. Energy restriction in obese subjects impact differently two mitochondrial function markers. J Physiol Biochem. 2008;64:211-9.

37. Linna MS, Ahotupa M, Kukkonen-Harjula K, et al. Co-existence of insulin resistance and high concentrations of circulating oxidized LDL lipids. Ann Med. 2015;47:394-8.

38. Carvalho-Filho MA, Ueno M, Hirabara SM, et al. S-nitrosation of the insulin receptor, insulin receptor substrate 1 , and protein kinase B/Akt: a novel mechanism of insulin resistance. Diabetes 2005;54:959-67.

39. Espinosa A, Campos C, Díaz-Vegas A, et al. Insulin-dependent $\mathrm{H} 2 \mathrm{O} 2$ production is higher in muscle fibers of mice fed with a high-fat diet. Int J Mol Sci. 2013;14:15740-54.

40. Alam MN, Bristi NJ, Rafiquzzaman M. Review on in vivo and in vitro methods evaluation of antioxidant activity. Saudi Pharm J. 2013;21:143-52.

41. Giampieri F, Tulipani S, Alvarez-Suarez JM, et al. The strawberry: composition, nutritional quality, and impact on human health. Nutrition. 2012;28:9-19.

42. Aaby K, Mazur S, Nes A, et al. Phenolic compounds in strawberry (Fragaria $x$ ananassa Duch.) fruits: Composition in 27 cultivars and changes during ripening. Food Chem. 2012;132:86-97.

43. Alvarez-Suarez JM, Giampieri F, Tulipani S, et al. One-month strawberry-rich anthocyanin supplementation ameliorates cardiovascular risk, oxidative stress markers and platelet activation in humans. J Nutr Biochem. 2014;25:289-94.

44. Tulipani S, Armeni T, Giampieri F, et al. Strawberry intake increases blood fluid, erythrocyte and mononuclear cell defenses against oxidative challenge. Food Chem. 2014;156:87-93.

45. Cao G, Russell RM, Lischner N, et al. Serum antioxidant capacity is increased by consumption of strawberries, spinach, red wine or vitamin C in elderly women. J Nutr. 1998;128:2383-90.

46. Edirisinghe I, Banaszewski K, Cappozzo J, et al. Strawberry anthocyanin and its association with postprandial inflammation and insulin. $\mathrm{Br} \mathrm{J}$ Nutr. 2011;106:913-22.

47. Burton-Freeman B, Linares A, Hyson D, et al. Strawberry modulates LDL oxidation and postprandial lipemia in response to high-fat meal in overweight hyperlipidemic men and women. J Am Coll Nutr, 2010;29:46-54

48. Zunino SJ, Parelman MA, Freytag TL, et al. Effects of dietary strawberry powder on blood lipids and inflammatory markers in obese human subjects. Br J Nutr. 2012;108:900-9.

49. Basu A, Betts NM, Nguyen A, et al. Freeze-dried strawberries lower serum cholesterol and lipid peroxidation in adults with abdominal adiposity and elevated serum lipids. J Nutr. 2014;144:830-7.

50. Ellis CL, Edirisinghe I, Kappagoda T, et al. Attenuation of meal-induced inflammatory and thrombotic responses in overweight men and women after 6-week daily strawberry (Fragaria) intake. A randomized placebo-controlled trial. J Atheroscler Thromb. 2011;18:318-27.

51. Devore EE, Kang JH, Breteler MMB, et al. Dietary intakes of berries and flavonoids in relation to cognitive decline. Ann Neurol. 2012;72:135-43.

52. Joseph JA, Shukitt-Hale B, Willis LM. Grape juice, berries, and walnuts affect brain aging and behavior. J Nutr. 2009;139:1813-7.

53. Vauzour D. Effect of flavonoids on learning, memory and neurocognitive performance: Relevance and potential implications for Alzheimer's disease pathophysiology. J Sci Food Agric. 2014;94:1042-56. 
54. Cassidy A, Mukamal KJ, Liu L, et al. High anthocyanin intake is associated with a reduced risk of myocardial infarction in young and middle-aged women. Circulation. 2013;127:188-96.

55. Pieszka M, Tombarkiewicz B, Roman A, et al. Effect of bioactive substances found in rapeseed, raspberry and strawberry seed oils on blood lipid profile and selected parameters of oxidative status in rats. Environ Toxicol Pharmacol. 2013;36:1055-62.

56. Diamanti J, Mezzetti B, Giampieri F, et al. Doxorubicin-induced oxidative stress in rats is efficiently counteracted by dietary anthocyanin differently enriched strawberry (Fragaria $\times$ ananassa Duch.). J Agric Food Chem. 2014;62:3935-43.

57. Kanodia L, Borgohain M, Das S. Effect of fruit extract of Fragaria vesca L. on experimentally induced inflamatory bowel disease in albino rats. Indian J Pharmacol. 2011;43:18-21.
58. Kosmala M, Zduńczyk Z, Kołodziejczyk K, et al. Chemical composition of polyphenols extracted from strawberry pomace and their effect on physiological properties of diets supplemented with different types of dietary fibre in rats. Eur J Nutr. 2014;53:521-32.

59. Casto BC, Knobloch TJ, Galioto RL, et al. Chemoprevention of ora cancer by lyophilized strawberries. Anticancer Res. 2014;33:4757-66.

60. Dudonné S, Dubé P, Pilon G, et al. Modulation of strawberry/cranberry phenolic compounds glucuronidation by co-supplementation with onion: characterization of phenolic metabolites in rat plasma using an optimized SSPE-UHPLC-MS/MS method. J Agric Food Chem. 2014;62:3244-56.

61. Saha J, Debnath M, Saha A, et al. Response surface optimisation of extraction of antioxidants from strawberry fruit, and lipid peroxidation inhibitory potential of the fruit extract in cooked chicken patties. J Sci Food Agric. 2011;91:1759-65. 